\title{
Application of double layered end-to-end anastomosis with continuous manual suture for completing digestive tract reconstruction in totally laparoscopic distal gastrectomy
}

XinSheng Zhang ${ }^{1}$, WeiBin Zhang ${ }^{2}$, MengLang Yuan ${ }^{1}$, XiaoMeng Shi ${ }^{1}$, HongYi Chen ${ }^{1}$, Zhen Feng ${ }^{1}$, ZiHao Chen ${ }^{1}$, DunBo Liu', EnJun Yan ${ }^{1}$ and ShuangYi Ren ${ }^{1 *}$ (1)

\begin{abstract}
Background: We retrospectively reviewed and consecutively collected the clinical data of distal gastric cancer patients who received surgical treatment, and we discuss the safety and feasibility of double layered end-to-end anastomosis with continuous manual suture to complete digestive tract reconstruction in totally laparoscopic distal gastrectomy.

Methods: We reviewed the clinical data of 41 patients with distal gastric cancer from the gastroenterology department of the Second Affiliated Hospital of Dalian Medical University, from September 2018 to August 2019, who underwent totally laparoscopic distal gastrectomy. During the operation, the method of double layered end-to-end anastomosis with continuous manual suture was used for Billroth type I anastomosis to complete digestive tract reconstruction. All patients have been given a follow-up visit and gastroscopy three months after the operation. The peri-operative clinical information and postoperative follow-up information were collected for analysis, and the clinical application value was evaluated.

Results: General information: male $(n=27)$, female $(n=14)$, age $=65.02($ SD 9.94) years, and BMI $=23.52(S D 2.56) \mathrm{kg} /$ $\mathrm{m}^{2}$, Tumor location: antrum(32,78.0\%), angle (6,14.6\%), and body (3,7.3\%). Clinical stage: I (27,65.9\%), II (7, 17.1\%), and III (7, 17.1\%). Operative information: operation time $=154.51$ (SD 33.37) $\mathrm{min}$, anastomosis time $=26.88($ SD 5.11) $\mathrm{min}$; intraoperative bleeding $=66.34(\mathrm{SD} 48.81) \mathrm{ml}$; first postoperative ambulation Median $=1(\mathrm{IQR} 0) \mathrm{d}$, first postoperative flatus Median =3(IQR 2) d, first postoperative diet Median =3(IQR 1) d, postoperative hospital stay Median =7(IQR 2) $d$, and total hospitalization cost $=10,935.00(S D$ 2205.72) USD. Differentiation degree: high and high-moderate $(3,7.32 \%)$, moderate and poor-moderate $(24,58.54 \%)$, poor differentiation $(14,34.15 \%)$, dissected lymph nodes Median $=31$ (IQR 17), and positive lymph nodes Median =0(IQR 1). Pathological stage: IA (20,48.78\%), IB (3, 7.32\%), IIA (4, 9.76\%), IIB (5, 12.20\%), IIIA (1, 2.44\%), IIIB (3, 7.32\%), and IIIC (5, 12.20\%). Complications $(n=4)$ : lung infection (1, $2.44 \%)$, anastomotic leakage $(1,2.44 \%)$, and gastroparesis $(2,4.88 \%)$.
\end{abstract}

\footnotetext{
*Correspondence: rsydl@aliyun.com

${ }^{1}$ Department of General Surgery, The Second Affiliated Hospital of Dalian

Medical University, Zhongshan Road 467, Shahekou District, Dalian,

Liaoning, China

Full list of author information is available at the end of the article
}

(C) The Author(s) 2021. Open Access This article is licensed under a Creative Commons Attribution 4.0 International License, which permits use, sharing, adaptation, distribution and reproduction in any medium or format, as long as you give appropriate credit to the original author(s) and the source, provide a link to the Creative Commons licence, and indicate if changes were made. The images or other third party material in this article are included in the article's Creative Commons licence, unless indicated otherwise in a credit line to the material. If material is not included in the article's Creative Commons licence and your intended use is not permitted by statutory regulation or exceeds the permitted use, you will need to obtain permission directly from the copyright holder. To view a copy of this licence, visit http://creativecommons.org/licenses/by/4.0/. The Creative Commons Public Domain Dedication waiver (http://creativeco mmons.org/publicdomain/zero/1.0/) applies to the data made available in this article, unless otherwise stated in a credit line to the data. 
Conclusion: It is safe and feasible in clinical treatment to apply the method of double layered end-to-end anastomosis with continuous manual suture to complete digestive tract reconstruction in totally laparoscopic distal gastrectomy.

Keywords: Distal gastric cancer, Continuous manual suture, Digestive tract reconstruction, Totally laparoscopic distal gastrectomy, Double layered end-to-end anastomosis with continuous manual suture

\section{Background}

In recent years, with the advanced development of laparoscopic techniques, totally laparoscopic gastrectomy has been suggested to be safe and feasible, especially, totally laparoscopic distal gastrectomy (TLDG) [1]. The choice of the ideal method for digestive tract reconstruction in distal gastrectomy is still controverial. Various methods of anastomosis with a stapler have been heavily reported in previous studies, such as using linear staplers to perform delta-shaped anastomosis, Billroth II anastomosis or Roux-en-Y anastomosis [2-4]. However, there are still many problems, such as high anastomotic tension, inaccurate tumour margins and large expenses. Therefore, we started to perform totally laparoscopic hand-sewn Billroth I anastomosis with a new application of double layered end-to-end anastomosis with continuous manual suture to complete digestive tract reconstruction, and we have completed 41 cases thus far. We intend to analyse these cases and discuss the relevant issues about of this the new application in totally laparoscopic distal gastrectomy based on previously published studies and our own experiences.

\section{Methods \\ Materials}

We retrospectively reviewed and consecutively collected the clinical data of 41 patients with distal gastric cancer from the gastroenterology department of the Second Affiliated Hospital of Dalian Medical University, from September 2018 to August 2019, who underwent totally laparoscopic distal gastrectomy. During the operation, the method of double layered end-to-end anastomosis with continuous manual suture was used for Billroth I type anastomosis to complete digestive tract reconstruction.

\subsection{Inclusion criteria}

(1) Electronic gastroscopy and biopsy pathological diagnoses were used to confirm distal gastric cancer before surgery; (2) Chest and abdominal computed tomography (CT) scans confirmed no distant metastasis before surgery; and (3) No serious complications of the heart, lungs or other important organs.

\subsection{Exclusion criteria}

(1) Severe cardiopulmonary cerebral dysfunction, resistant to general anaesthesia and surgery: refractory hypertension, cardiopulmonary dysfunction, respiratory failure, chronic obstructive pneumonia etc.; and (2) Severe liver, renal and coagulation dysfunction; (3) Other operation methods.The operation was performed by the same senior doctors. The patients and their families signed informed consent forms related to the operation. This study was approved by the Institutional Review Board of The Second Affiliated Hospital of Dalian Medical University.

\section{Methods}

2.1. No gastric tubes were placed before any of the operations. All the operations were performed using the German Braun AESCULAP 3D HD laparoscopic system. The anesthesia method is intravenous-inhalation combined anesthesia. The operation position is head high and foot low (tilt $15^{\circ}$ ). And trocar position of the puncture are shown in Fig. 1. According to the 15th edition of the Japanese Classification of Gastric Carcinoma, laparoscopic distal gastrectomy and D2 lymph node dissection were performed.

2.2. Digestive tract reconstruction.

\subsubsection{Anastomotic placement.}

The duodenum and the greater curvature of the remnant stomach were occluded with interdiction clamps located approximately $2 \mathrm{~cm}$ away from the predetermined position of the anastomotic opening to block the reflux of digestive fluid.

2.2.2 The first single needle for continuous suture of the sarcoplasmic layer of the posterior wall.

First, if necessary, the upper and lower margins of the posterior duodenal wall were sewn with one stitch each to the seromuscular layer of the posterior gastric wall to obtain relative immobilization between the duodenal stump and the remnant stomach, Then, the posterior wall of the remnant stomach and the posterior wall of the duodenum were sutured contin- 


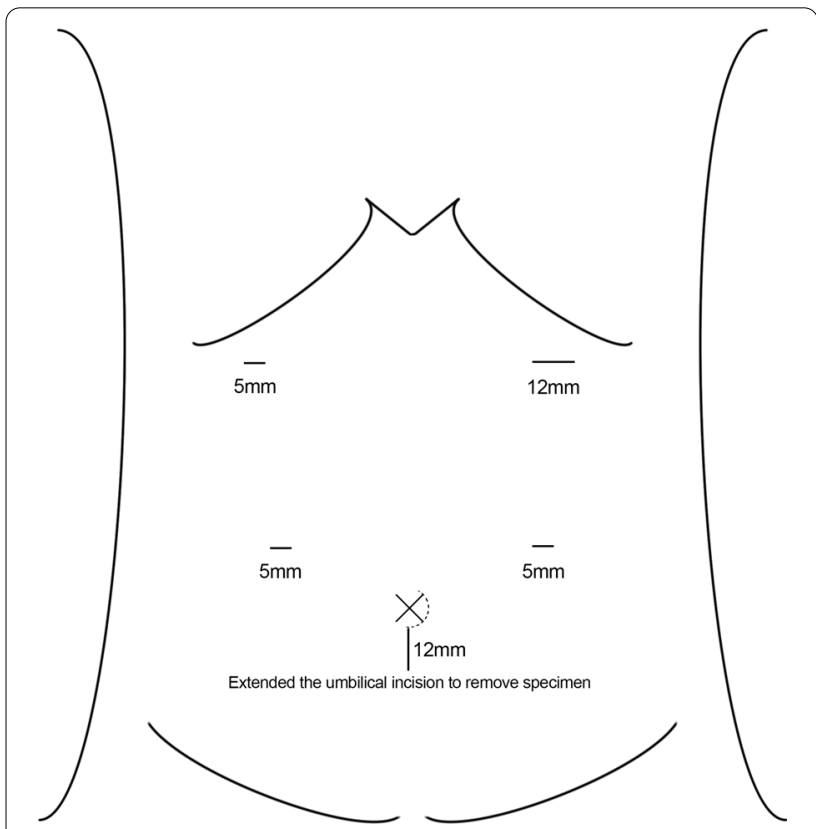

Fig. 1 Placement of trocars. The figure depicted in Fig. 1 was drawn by our own. Two $12 \mathrm{~mm}$ and three $5 \mathrm{~mm}$ trocars were used in operation. The $12 \mathrm{~mm}$ trocar below the umbilicus is used as the observation hole, and another $12 \mathrm{~mm}$ trocar is the main operation hole

uously from top to bottom with an absorbable barbed thread with single needle (Covidien VLOCL0614, $3-0)$, and the needle was kept for standby.

2.2.3. The second single needle for continuous lockstitch suture of the whole layer from the posterior wall to the anterior wall.

The whole layer of the duodenal stump and the remnant stomach were cut through with ultrasonic dissector, and those sizes were equal to the width of the duodenum, which were as the two ends of the anastomosis. The second absorbable barbed thread with single needle (Covidien VLOCL0614, 3-0) was used for continuous lock-stitch suture, from bottom to top, of the whole layer of the posterior wall of the anastomosis. Then, the Connell suture method was adopted to sew the whole layer of the anterior wall of the anastomosis until the starting point of the second single needle, and the anastomosis was completely closed.

2.2.4 The first standby needle for continuous suturing of the sarcoplasmic layer of the anterior wall.

From bottom to top, the sarcoplasmic layer anastomosis of the anterior wall was continuously sutured with the first standby needle to completely embed the sarcoplasmic layer of the anastomosis.

2.2.5 Release of the interdiction clamps.
The interdiction clamps were released on both sides of the anastomosis, and the reconstruction of the digestive tract was completed. If necessary, an electronic gastroscope was used to check the internal patency of the anastomosis and whether there were bleeding or weak points.

See Fig. 2 for details.

\section{Observation index}

3.1. General information: age, sex, BMI, previous abdominal surgery, tumor location, and clinical stage.

3.2. Surgical information: operation time, anastomosis time, intraoperative bleeding; tumour diameter, tumor margin, dissected lymph node, first postoperative ambulation, first postoperative flatus, first postoperative diet, postoperative hospital stay, total hospitalization cost, differentiation degree, pathological stage, and complications.

3.3. Postoperative follow-up information: we performed an upper gastrointestinal imaging at 6 days, an upper gastrointestinal imaging and a gastroscopy at 1 month, and a gastroscopy at 3 months after the operation in all of the patients.

\section{Fitting equation and CUSUM analysis}

All patients were arranged according to the operation sequence and calculated according to the equation: CUSUM $=\sum_{\mathrm{i}=1}^{\mathrm{n}}(\mathrm{Xi}-\mu)$, Xi represents the operation time or anastomosis time of each patient, $\mu$ represents the corresponding mean time, and $\mathrm{n}$ represents the serial number of the patient.

Draw scatter diagram of learning curve: the operation sequence as horizontal axis and CUSUM value as vertical axis. SPSS 24.0 statistical software was used to fit the learning curve. The fitting model test was based on $P$ value, $P<0.05$ as the standard of curve fitting success. The goodness of fit is determined by coefficient $R^{2}$, that is, the closer $R^{2}$ is to 1 , the higher the goodness of fit is, and the model with the largest $R^{2}$ is selected as the best fitting model. The falling point in the curve represents the starting point of the case data lower than the mean value, and the corresponding horizontal axis is the number of surgical cases needed to cross the learning curve.

\section{Statistical method}

All data analysis was performed using SPSS 24.0 statistical software. The count data were measured by $X^{2}$ tests, the normal distribution measurement data were described $a s^{-} x \pm s$, and the skewed distribution 
(See figure on next page.)

Fig. 2 Steps of double layered end-to-end anastomosis with continuous manual suture to complete digestive tract reconstruction in TLDG (Video capture of anastomotic procedure and sketch of anastomosis). a The first absorbable barbed thread with single needle(Covidien VLOCL0614, 3-0) to continuous suture of sarcoplasmic layer of the posterior wall from the top to the bottom, and the needle was kept for standby. $\mathbf{b}$ The second absorbable barbed thread with single needle (Covidien VLOCL0614, 3-0) to continuous lock-stitch suture, from bottom to top, of the whole layer from the posterior wall to anterior wall. c The second needle to Connell suture of the whole layer of the anterior wall of the anastomosis. $\mathbf{d}$ The first standby needle to continuous suture of sarcoplasmic layer of the anterior wall. e Display of anastomotic anterior wall. f Display of anastomotic posterior wall.

measurement data were described as the Median (IQR). The standard level $\alpha$ was 0.05 , and the difference was statistically significant when $p<0.05$.

\section{Results}

\section{General information}

Our records identified though database searching from hospital's case retrieval system, according to those search conditions: 1. confirm distal gastric cancer preoperation; 2.no distant metastasis preoperation; 3.undergoing totally laparoscopic distal gastrectomy; 4.from Sep 2018 to Aug 2019. The total number of patients undergoing totally laparoscopic distal gastrectomy in the study period was 111.70 cases of those that did not meet the requirements were excluded one by one, according to the exclusion criteria: refractory hypertension, cardiopulmonary dysfunction, respiratory failure, chronic obstructive pneumonia, renal insufficiency, other operation method. Finally, 41 cases were included. A flow diagram has been added(Fig. 3).

A total of 41 patients were included in this study: 27 males and 14 females. The mean age was $(65.02 \pm 9.94)$ years. The mean BMI was $(23.52 \pm 2.56) \mathrm{kg} / \mathrm{m}^{2}$. The tumour location was distributed as follows: antrum (32, $78.0 \%)$, angle $(6,14.6 \%)$, and body $(3,7.3 \%)$. The tumour clinical stage was distributed as follows: I (27, 65.9\%), II (7, 17.1\%), III (7, 17.1\%).

As shown in Table 1.

\section{Surgical Information}

The operations were smooth, and no serious complications occurred in the perioperative period.

The mean duration of the operation was $(154.51 \pm 33.37) \mathrm{min}$ and the mean time to complete the anastomosis was $(26.88 \pm 5.11) \mathrm{min}$, as shown in Fig. 4. The amount of intraoperative bleeding was $(66.34 \pm 48.81) \mathrm{ml}$, and the mean tumor diameter was $(3.07 \pm 1.68) \mathrm{cm}$. The proximal tumor margin was $5.09 \pm 1.47 \mathrm{~cm}$. The distal tumour margin was $(3.23 \pm 1.28) \mathrm{cm}$. The number of dissected lymph nodes was Median =31(17), and the number of positive lymph nodes was Median $=0$ (1). The differentiation degree was distributed as follows: high and high-moderate $(3,7.32 \%)$, moderate and poor-moderate $(24,58.54 \%)$, and poor differentiation (14, 34.15\%). In terms of the postoperative pathological TNM stage, the results showed that there were 23 patients in stage I: IA (20, 48.78\%), and IB (3, 7.32\%); 9 patients in stage II: IIA $(4,9.76 \%)$ and IIB (5, $12.20 \%)$; and 9 patients in stage III: IIIA (1, 2.44\%), IIIB (3, 7.32\%), IIIC $(5,12.20 \%)$.

Of the 41 patients, no serious complications occurred, but four controllable complications occurred: one case of postoperative pulmonary infection $(1,2.44 \%)$, one case of anastomotic leakage $(1,2.44 \%)$, and two cases of gastroparesis $(2,4.88 \%)$.According to Clavien Dindo classification, postoperative complications were evaluated, including Grade I(3, 7.3\%), Grade II(1, 2.4\%). All the above patients recovered after conservative nonoperative treatment.

All patients were able to get out of bed the first day after the operation. The first postoperative ambulation occurred at Median $=1(0)$ days, and the first postoperative flatus occurred at Median $=3(2)$ days. The first postoperative diet occurred at Median $=3(1)$ days. The postoperative hospital stay was Median $=7(2)$ days, and the total hospitalization cost was 10,935.00 \pm 2205.72 USD.

As shown in Table 2.

\section{Postoperative follow-up information}

All the patients returned for visits in the outpatient department during the 1st and 3rd months after the operation. All patients exhibited a satisfactory appetite, and there were no symptoms of discomfort such as abdominal pain or bloating. Upper gastrointestinal imaging showed that the anastomosis was unobstructed, as the contrast agent smoothly entered the duodenum (Fig. 5). Gastroscope examination 3 months after the operation showed that the anastomosis had healed well and that there was no stenosis (Fig. 6).

\section{CUSUM analysis}

The best fitting equation of CUSUM learning curve was: CUSUM (operation time $)=-\quad 1.52+21.72 * \mathrm{X}-0.52 * \mathrm{X}^{2}-1.19^{*} 10^{-}$ ${ }^{3 *} \mathrm{X}^{3}, \quad \mathrm{CUSUM}(\quad$ anastomosis time $)=-15+8.52 * X-0.24 * X^{2}+9.81^{*} 10^{-4 *} X^{3} \quad(\quad X$ 

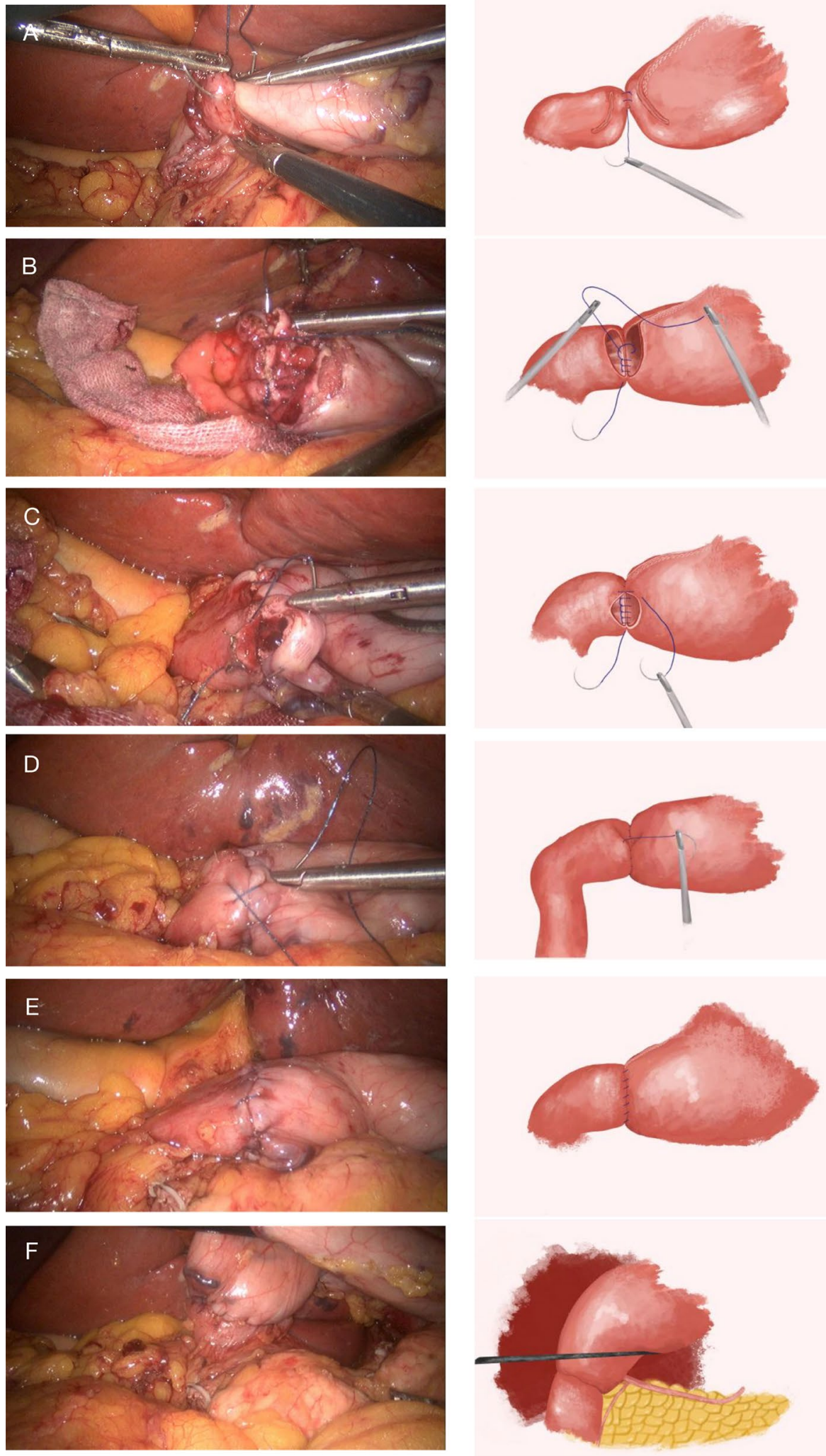
(See figure on next page.)

Fig. 3 A flow diagram for the study selection. Our records identified though database searching from hospital's case retrieval system, according to the search conditions: 1. Confirm distal gastric cancer preoperation; 2.No distant metastasis preoperation; 3.Undergoing totally laparoscopic distal gastrectomy; 4.From Sep 2018 to Aug 2019. The total number of patients undergoing totally laparoscopic distal gastrectomy in the study period was 111.70 cases of those that do not meet the requirements were excluded one by one, according to the exclusion criteria: refractory hypertension, cardiopulmonary dysfunction, respiratory failure, chronic obstructive pneumonia etc., severe liver, renal and coagulation dysfunction, and other operation method. Finally, 41 cases were included

means surgical cases), the $\mathrm{P}$ value of fitting model test were $<0.05$, with goodness of fit $\left(R^{2}\right)$ as 1 . CUSUM (operation time) reached the peak when the number of surgical cases accumulated to the 21th case, and 21 cases were the minimum number of surgeries needed to cross the learning curve. As the same way, CUSUM (anastomosis time) reached the peak when the number of surgical cases accumulated to the 21th case, and 21cases were the minimum number of surgeries needed to skillfully master double layered end-to-end anastomosis with continuous manual suture to complete digestive tract reconstruction across the learning curve(Fig. 7).

\section{Discussion}

Totally laparoscopic gastrectomy has shown distinct advantages compared to laparoscopic-assisted gastrectomy [4-6]. In totally laparoscopic distal gastrectomy, digestive tract reconstruction has been a key and difficult part of surgery. At present, various anastomotic methods have their own advantages and disadvantages. For patients with lower gastric cancer, most of them undergo delta-shaped anastomosis, Billroth II anastomosis or Roux-en-Y anastomosis with a stapler [1, 7 , 8]. Billroth I anastomosis is considered to be more in line with human physiology and anatomy, so it has been favour by surgeons. To date, triangle-shaped anastomosis or modified triangle-shaped anastomosis with a stapler is the most commonly chosen kind of Billroth I anastomosis [7, 9]. Totally laparoscopic triangle-shaped anastomosis with a stapler requires overlapping the remnant stomach wall and duodenum, theoretically wasting part of the remnant stomach wall and duodenal wall and increasing the anastomotic tension. At the same time, the dissection of tumours located higher or near the gastric body is prone to causing insufficient margins, thus greatly limiting the indications for totally laparoscopic Billroth I anastomosis [10]. Billroth II anastomosis can lead to reflux gastritis due to bile reflux, which can increase the incidence of remnant gastric cancer [11]. As a result, this procedure has fallen out of favour for digestive tract reconstruction. Roux-en-Y anastomosis requires two anastomoses, thus increasing the number of intestinal stumps, and requires rather complicated steps. Additionally, similar to Billroth II anastomosis, Roux-en-Y anastomosis does not highly conform to human physiology and anatomy $[8,12]$. If postoperative complications of biliary tract diseases such as bile duct stones occur, ERCP and other tests cannot be performed. Totally laparoscopic handsewn Billroth I anastomosis can avoid the shortcomings of both Roux-en-Y anastomosis and Billroth II anastomosis.

\section{Indications}

At present, there remain no published studies at home or abroad on hand-sewn Billroth I anastomosis for digestive tract reconstruction after totally laparoscopic distal gastrectomy for the treatment of lower gastric cancer. Our team developed a hand suture technique to directly perform standard end-to-end anastomosis of the remnant stomach and duodenal stump. Similar to hand-sewn Billroth I anastomosis in open gastrectomy, this method could reserve partial walls of the remnant stomach and duodenum compared with triangle-shaped anastomosis with a stapler, ensuring sufficient tumour margins and radical dissection of tumour tissues. Therefore, operative indications for totally laparoscopic Billroth I anastomosis can be greatly broadened, and the difficulty of the surgery can ultimately be reduced. Our team concluded that indications for totally laparoscopic hand-sewn Billroth I anastomosis should be equivalent to those for traditional Billroth I anastomosis in open gastrectomy under the mature cooperation of skilled surgeons.

\section{Safety and feasibility}

In our study, the mean duration of the operation was $154.51 \pm 33.37 \mathrm{~min}$ and the mean time to complete the anastomosis was $26.88 \pm 5.11 \mathrm{~min}$ (Fig. 4). The amount of intraoperative bleeding was $66.34 \pm 48.81 \mathrm{~mL}$. These data indicate that manual reconstruction of digestive tract is feasible [13]. Lymph node dissection was performed strictly according to standard radical (D2) lymphadenectomy. The pathological examination of the proximal and distal margins of the specimens was negative, and the tumour-free distance of both proximal and distal margins was within the required range (Table 2). Therefore, radical dissection could be guaranteed. Gastrointestinal imaging showed normal gastric emptying on the 6th 
Records identified though database searching from hospital's case retrieval system
1. Confirm distal gastric cancer preoperation

2.No distant metastasis preoperation

3.Undergoing totally laparoscopic distal gastrectomy

4.From Sep 2018 to Aug 2019

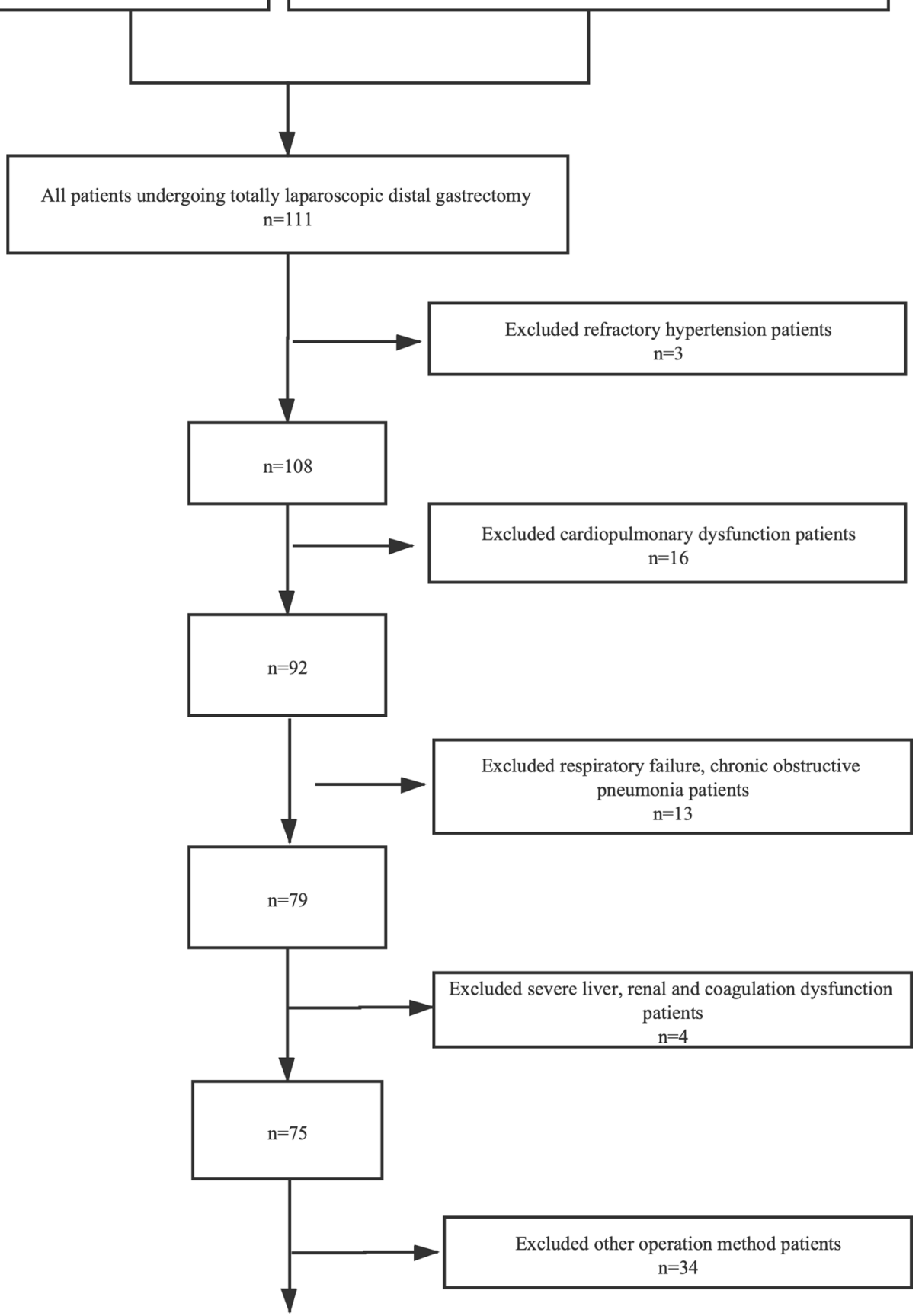

The method of double layered end-to-end anastomosis with continuous manual suture

$\mathrm{n}=41$ 
Table 1 General Information

\begin{tabular}{ll}
\hline Index & $\mathbf{n = 4 1}$ \\
\hline Age(years) & $65.02 \pm 9.94$ \\
Sex(M/F) & $27 / 14$ \\
BMI(kg/m²) & $23.52 \pm 2.56$ \\
Previous abdominal surgery(n) & $2(13.3)$ \\
Tumor location & \\
Antrum & $32(78.0)$ \\
Angle & $6(14.6)$ \\
Body & $3(7.3)$ \\
Clinical stage & \\
I & $27(65.9)$ \\
II & $7(17.1)$ \\
III & $7(17.1)$ \\
IV & 0 \\
\hline
\end{tabular}

The data were expressed as mean \pm SD or number (\%)

$F$ female, $M$ male

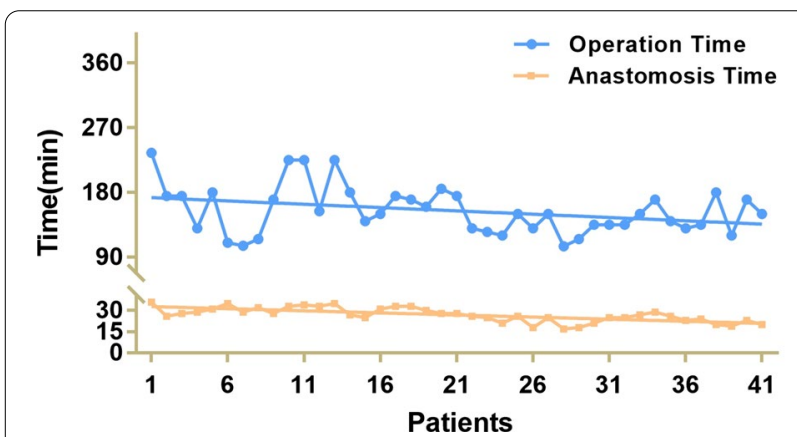

Fig. 4 Time of the total operation and the hand-sewn anastomosis of each patient. The hand-sewn anastomosis procedural time was defined as the time from suturing the seromuscular layer of posterior duodenal wall and gastric wall to the seromuscular suture completed of the anterior wall of anastomotic stoma

postoperative day (Fig. 5). Only 1 patient developed pulmonary infection and received anti-infective treatment. One patient developed anastomotic leakage, received unobstructed surgical drainage, received placement of a nasoenteral nutrition tube for enteral nutrition support, and recovered 2 weeks later. There were two patients with gastroparesis who recovered on the 18th and 25th days after the operation by gastrointestinal decompression and nasoenteral nutrition tube placement through $\mathrm{s}$ gastroscope for enteral nutrition. All the above patients recovered after conservative nonoperative treatment. All 41 patients recovered well and were followed after surgery, and no serious complications or perioperative death occurred.

The patients returned for a visit on the 3rd month after surgery, and the gastroscopic results showed
Table 2 Intraoperative and postoperative condition [means \pm standard deviations, Median (IQR), n (\%)]

\begin{tabular}{|c|c|}
\hline Index & $\mathrm{n}=41$ \\
\hline Operation time (min) & $154.51 \pm 33.37$ \\
\hline Anastomosis time ${ }^{\mathrm{a}}(\mathrm{min})$ & $26.88 \pm 5.11$ \\
\hline Intraoperative bleeding (ml) & $66.34 \pm 48.81$ \\
\hline Maximal tumor diameter (cm) & $3.07 \pm 1.68$ \\
\hline Proximal tumor margin (cm) & $5.09 \pm 1.47$ \\
\hline Distal tumor margin (cm) & $3.23 \pm 1.28$ \\
\hline Dissected lymph node (Median) & $31(17)$ \\
\hline Positive lymph node (Median) & $0(3)$ \\
\hline First postoperative ambulation (Median) & $1(0)$ \\
\hline First postoperative flatus (Median) & $3(2)$ \\
\hline First postoperative diet (Median) & $3(1)$ \\
\hline Postoperative hospital stay (Median) & $7(2)$ \\
\hline Total hospitalization cost (USD) & $10,935.00 \pm 2205.72$ \\
\hline \multicolumn{2}{|l|}{ Differentiation degree } \\
\hline High and high-moderate differentiation & $3(7.32)$ \\
\hline Moderate and poor-moderate differentiation & $24(58.54)$ \\
\hline Poor differentiation & $14(34.15)$ \\
\hline \multicolumn{2}{|l|}{ Pathological stage } \\
\hline IA & $20(48.78)$ \\
\hline IB & $3(7.32)$ \\
\hline$\| \mathrm{A}$ & $4(9.76)$ \\
\hline$\| \mathrm{B}$ & $5(12.20)$ \\
\hline$\| I I A$ & $1(2.44)$ \\
\hline$\| I \mid B$ & $3(7.32)$ \\
\hline IIIC & $5(12.20)$ \\
\hline IV & 0 \\
\hline \multicolumn{2}{|l|}{ Complications } \\
\hline Lung infection & $1(2.44)$ \\
\hline Anastomotic leakage & $1(2.44)$ \\
\hline Gastroparesis & $2(4.88)$ \\
\hline
\end{tabular}

The data were expressed as mean $\pm \mathrm{SD}$, Median(IQR)or number (\%), as appropriate

${ }^{\text {a }}$ The time from suturing the seromuscular layer of posterior duodenal wall and gastric wall to the seromuscular suture completed of the anterior wall of anastomotic stoma

unobstructed anastomosis and smooth mucosa (Fig. 6). In addition, the total hospitalization cost for this group of patients was $10,935.00 \pm 2205.72$ USD. Wang Y and others have reported that the total hospitalization cost was $14,784.00 \pm 2156.00$ USD [14, 15]. The total hospitalization cost can be roughly divided into these parts: In-patient examination fees( physical examination and Laboratory test fees), consumable material (anastomotic materials, ultrasonic knif, Trocars, Vicryl, etc.), medicine fees, hospitalization, physicians, nursing fees, etc. The price of anastomotic consumables is very expensive, $100 \%$ of the cost need to be paid by patients, accounting 

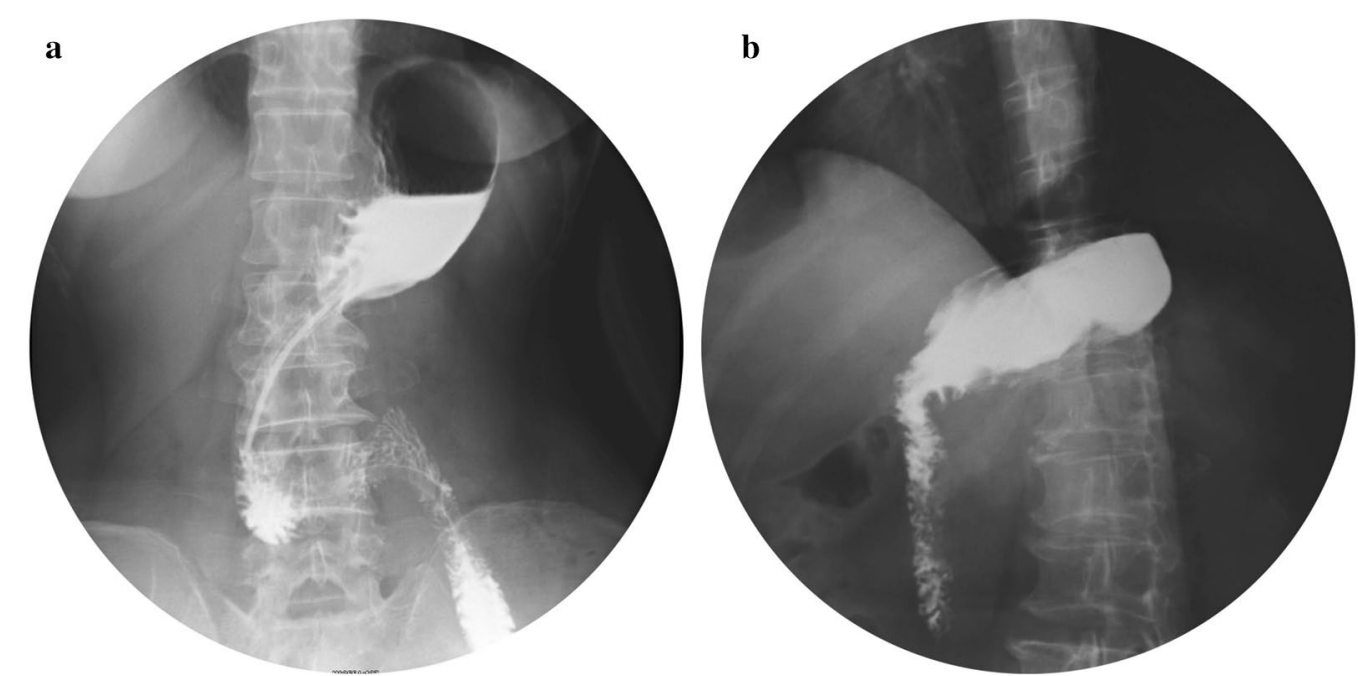

Fig. 5 Upper gastrointestinal imaging 6 days and 1 month after operation. a X-ray performed 6 days after the operation shows that the anastomotic stoma was unobstructed and there was no anastomotic leakage. $\mathbf{b}$ X-ray imaging performed 1 month after the operation
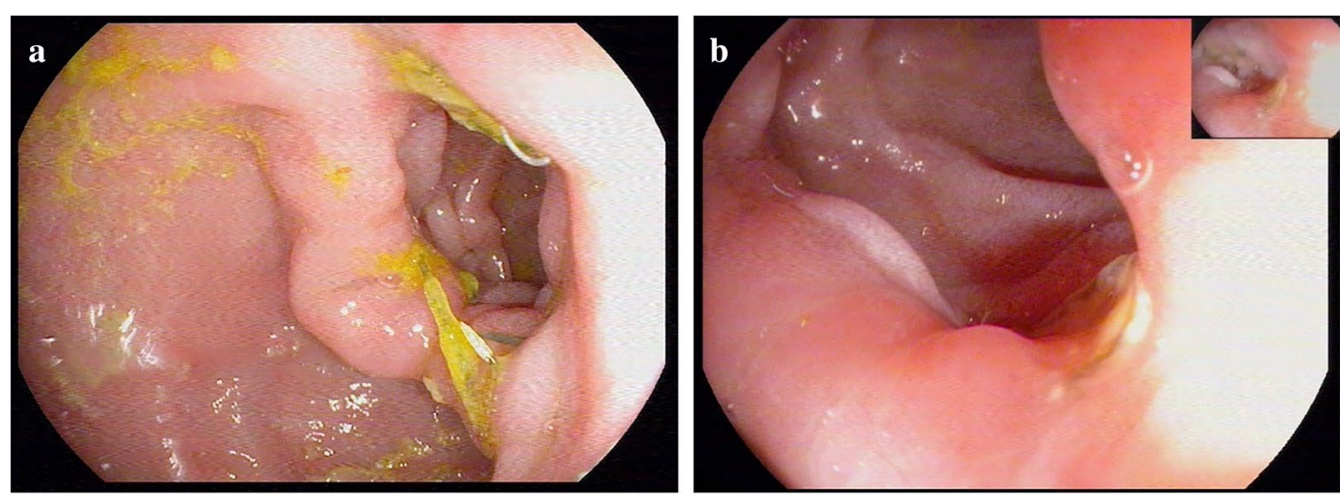

Fig. 6 Gastroscopic images of anastomosis 1 months and 3 months after the operation. a Gastroscopic view at postoperative 1 months after the hand-sewn anastomosis procedure. $\mathbf{b}$ Gastroscopic view at postoperative 3 months after the hand-sewn anastomosis procedure

for a large proportion of the total cost, while the other cost are in the scope of medical reimbursement, accounting for $60-90 \%$ of the reimbursement proportion. Therefore, saving the cost of anastomotic consumables can certainly reduce most of the cost and greatly reduce the economic pressure of patients. The results above indicate that totally laparoscopic hand-sewn Billroth I anastomosis is a safe and feasible method for digestive tract reconstruction.

\section{Advantages}

Totally laparoscopic hand-sewn Billroth I anastomosis has the following advantages. (1) It conforms to human anatomy and physiology. Totally laparoscopic hand-sewn
Billroth I anastomosis manages to save the continuity of the digestive tract and the feedback mechanism of autocrine and paracrine systems, which are more consistent with normal physiological structure. Therefore, reflux gastritis caused by bile reflux can be avoided [3]. (2) A clear field of vision during totally laparoscopic handsewn Billroth I anastomosis is guaranteed. Compared with other anastomotic methods, this method is limited to the right upper abdomen. There is no need to frequently change the field of vision, which can decrease the difficulty of assistant coordination. (3) The number of anastomoses is decreased, which may reduce complications such as anastomotic bleeding and leakage. Under the skilled cooperation of teamwork, the total time of the operation and time of anastomosis by hand 


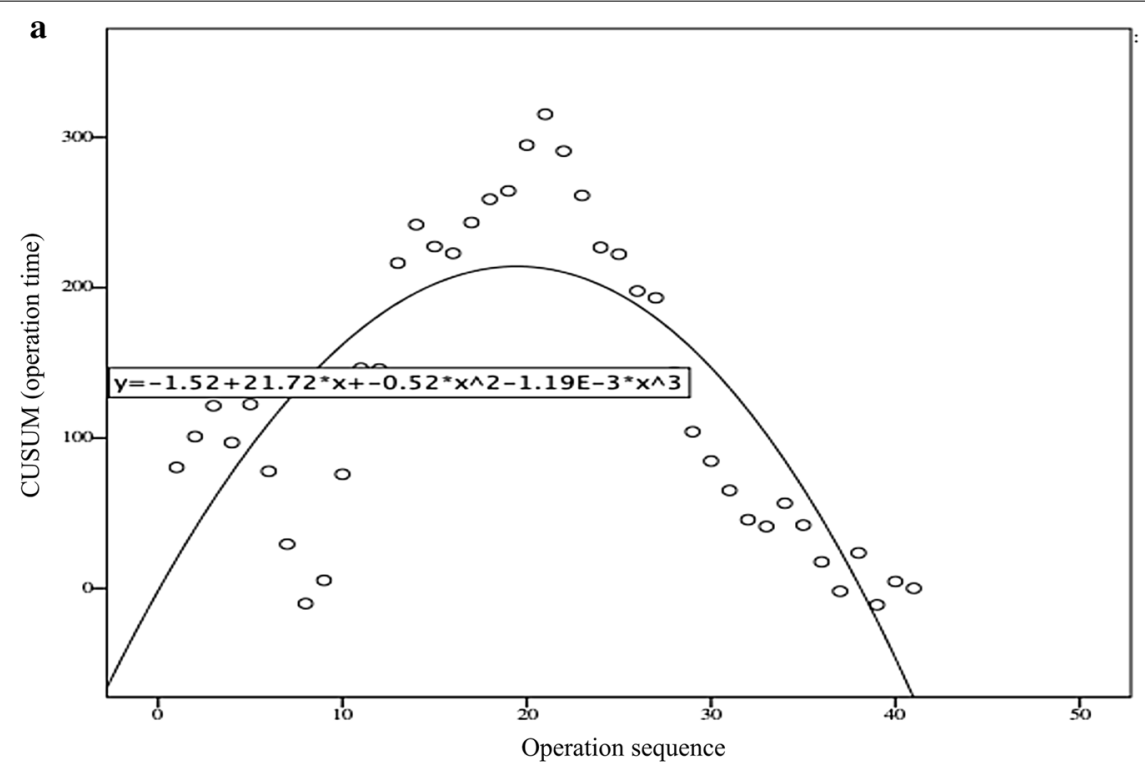

$\mathbf{R}^{2}$ Cubic $=0.638$

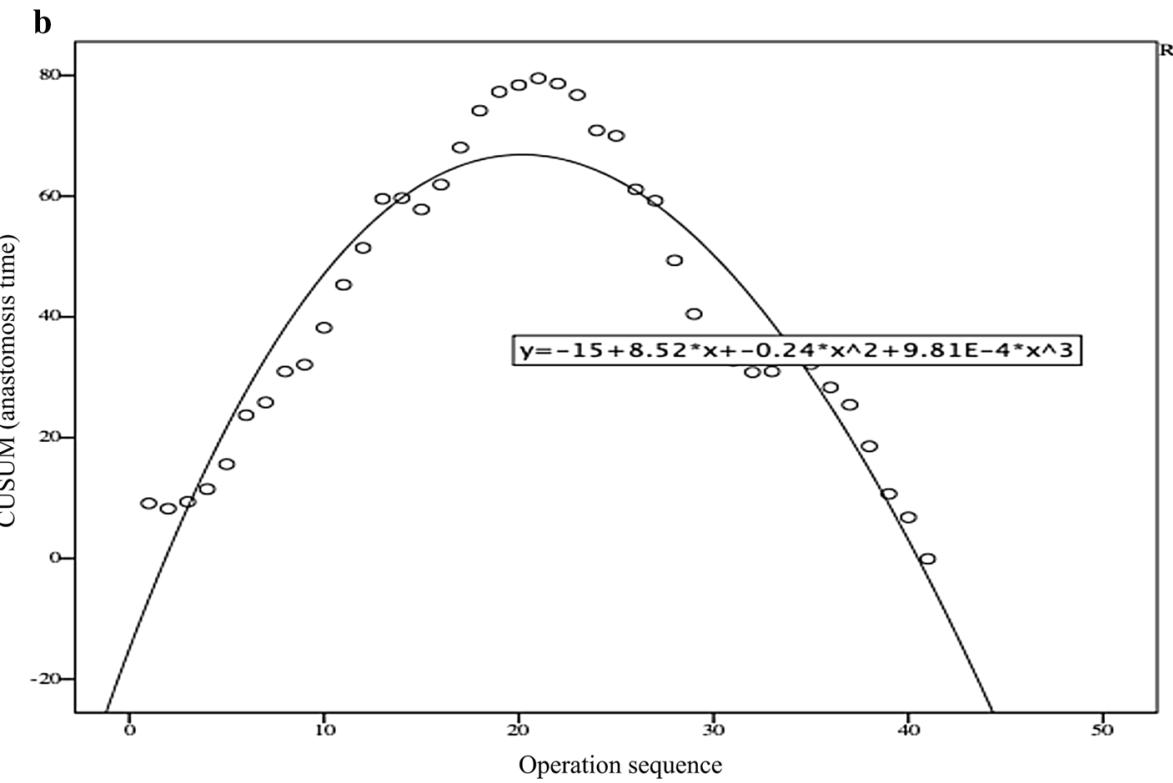

Fig. 7 CUSUM analysis for operation time and anastomosis time. The operation sequence as horizontal axis and CUSUM value as vertical axis. The fitting model test was based on $P$ value, $P<0.05$ as the standard of curve fitting success. The goodness of fit is determined by coefficient $R^{2}$, that is, the closer $R^{2}$ is to 1. a CUSUM analysis for operation time. $\mathbf{b}$ CUSUM analysis for anastomosis time

were acceptable [13]. In addition, the operation time was gradually reduced with the extension of the learning curve (Fig. 7). CUSUM analysis shows 21 cases were the minimum number of surgeries needed to skillfully master double layered end-to-end anastomosis with continuous manual suture to complete digestive tract reconstruction across the learning curve. (4) The hand-sewn anastomosis is relatively smooth compared to anastomosis with a stapler, which is prone to having overlapped corners or "dog ears", causing a higher risk of anastomotic leakage as well as adhesion. (5) The resection range and reduction in anastomotic tension are fully ensured. Hand-sewn Billroth I anastomosis is a standard end-to-end anastomosis, which can reserve the overlapped gastroduodenal wall wasted in triangle-shaped anastomosis with a stapler. (6) Mesenteric hiatal hernia and Petersen's hernia can be avoided in hand-sewn Billroth I anastomosis. (7) There are decreased complications such as adhesive ileus. This procedure is limited to the right upper abdomen, which can reduce potential mechanical stimulation and 
mucosal damage to the jejunum therefore decreasing the incidence rate of postoperative intestinal adhesion.

\section{Difficulties and countermeasures}

Although totally laparoscopic hand-sewn Billroth I anastomosis has many advantages, it is still a very difficult technique for some surgery teams that newly start practising totally laparoscopic hand-sewn Billroth I anastomosis. For example, it has been previously reported that intra-abdominal anastomosis can increase the incidence rate of abdominal infection [16]. In our study, a bulldog clamp was used to block the gastric and duodenal stumps to avoid intestinal fluid leakage, and this clamp was not removed until fullthickness anastomosis was completed. Additionally, the surgical team needs to discuss in advance the specific anastomosis steps in great detail to achieve perfect cooperation [17].

\section{Conclusions}

Double layered end-to-end anastomosis with continuous manual suture is a safe and feasible method for digestive tract reconstruction in totally laparoscopic distal gastrectomy, as it not only ensures the completion of Billroth I anastomosis but also may reduce the operation cost to a certain extent. It is worth further discussion and practice, and prospective controlled studies with large samples sizes are needed.

\section{Abbreviations}

TLDG: Totally Laparoscopic Distal Gastrectomy; CT: Computed Tomography.

\section{Acknowledgements}

Thanks to all the people, WB Z, ML Y, XM S, HY C, Z F, ZH C, DB L, EJ Y, who participated in the data collection, collation and statistics of the research: Thanks to Dr. SY R for his constructive revision of the research and his valuable comments, and thanks to the relevant departments of the hospital for their strong support for the research, such as general surgery, anesthesiology, imaging, laboratory, etc.Thank you very much.

\section{Authors' contributions}

All authors contributed to the study conception and design. Material preparation, data collection and analysis were performed by WB Z, MLY, XM S, HY C, Z F, ZH C, DB L, EJ Y. Dr. SY R summarized the overall treatment plan, designed the experimental ideas, checked the experimental data, calculated the statistical data. The first draft was written by Dr.XS Z and the previous versions of the manuscript were commented by all the other authors. The final manuscript was read and approved by all the authors.

\section{Funding}

This research was funded by National Natural Science Foundation of China with Grant No. 81372669, National Health Commission of the People's Republic of China with Grant No. W2012RQ23, and the Science and Technology Innovation Fund of Dalian City with grant No. 2018J13SN091; The funders had no role in the design of the study and collection, analysis, and interpretation of data and in writing the manuscript.

\section{Availability of data and materials}

The data analyzed during the current data-analysis is available from the corresponding author on reasonable request.

\section{Declarations}

Ethics approval and consent to participate

All procedures followed were in accordance with the ethical standards of the responsible committee on human experimentation (institutional and national) and with the Helsinki Declaration of 1964 and later versions.The study has been granted an exemption from the Ethics Committee of the Second Affiliated Hospital of Dalian Medical University Ethics Committee; Informed consent was signed with all individual participants included in the study.

\section{Consent for publication}

Not Applicable.

\section{Competing interests}

There is no conflict of interest in this research.

\section{Author details}

${ }^{1}$ Department of General Surgery, The Second Affiliated Hospital of Dalian Medical University, Zhongshan Road 467, Shahekou District, Dalian, Liaoning, China. ${ }^{2}$ General Surgery Department of Jinzhou Central Hospital, No.51, Section 2, Shanghai Road, Guta District, Jinzhou, Liaoning, China.

Received: 28 April 2020 Accepted: 14 April 2021

Published online: 26 April 2021

\section{References}

1. Xiao SM, Gao XJ, Zhao P. Comparison between total laparoscopy and laparoscopy-assisted distal gastrectomy for gastric cancer. A meta-analysis based on Japanese and Korean articles. Saudi Med J. 2014;35(11):1318-23.

2. Matsuhashi N, Yamaguchi K, Okumura N, Tanahashi T, Matsui S, Imai H, et al. The technical outcomes of delta-shaped anastomosis in laparoscopic distal gastrectomy: a single-center safety and feasibility study. Surg Endosc. 2017;31(3):1257-63.

3. Kim JH, Jun KH, Chin HM. Short-term surgical outcomes of laparoscopyassisted versus totally laparoscopic Billroth-II gastrectomy for gastric cancer: a matched-cohort study. BMC Surg. 2017;17(1):45.

4. Okuno K, Nakagawa M, Kojima K, Kanemoto E, Gokita K, Tanioka T, et al. Long-term functional outcomes of Roux-en-Y versus Billroth I reconstructions after laparoscopic distal gastrectomy for gastric cancer: a propensity-score matching analysis. Surg Endosc. 2018;32(11):4465-71.

5. Kumagai K, Hiki N, Nunobe S, Sekikawa S, Chiba T, Kiyokawa T, et al. Totally laparoscopic pylorus-preserving gastrectomy for early gastric cancer in the middle stomach: technical report and surgical outcomes. Gastric Cancer. 2015;18(1):183-7

6. Woo J, Lee JH, Shim KN, Jung HK, Lee HM, Lee HK. Does the difference of invasiveness between totally laparoscopic distal gastrectomy and laparoscopy-assisted distal gastrectomy lead to a difference in early surgical outcomes? A prospective randomized trial. Ann Surg Oncol. 2015;22(6):1836-43.

7. Wang SY, Hong J, Hao HK. A comparative study of delta-shaped and conventional Billroth I anastomosis after laparoscopic distal gastrectomy for gastric cancer. Surg Endosc. 2017;31(8):3191-202.

8. Resanovic A, Randjelovic T, Resanovic V, Toskovic B. Double tract vs. rouxen-Y reconstruction in the treatment of gastric cancer. Pak J Med Sci. 2018;34(3):643-8

9. Huang CM, Lin M, Lin JX, Zheng CH, Li P, Xie JW, et al. Comparision of modified and conventional delta-shaped gastroduodenostomy in totally laparoscopic surgery. World J Gastroenterol. 2014;20(30):10478-85.

10. Fukunaga T, Ishibashi Y, Oka S, Kanda S, Yube Y, Kohira Y, et al. Augmented rectangle technique for Billroth I anastomosis in totally laparoscopic distal gastrectomy for gastric cancer. Surg Endosc. 2018. https://doi.org/ 10.1007/s00464-018-6266-1. 
11. In Choi C, Baek DH, Lee SH, Hwang SH, Kim DH, Kim KH, et al. Comparison between billroth-II with braun and roux-en-Y reconstruction after laparoscopic distal gastrectomy. J Gastrointest Surg. 2016;20(6):1083-90.

12. Sun MM, Fan YY, Dang SC. Comparison between uncut RouX-en-Y and Roux-en-Y reconstruction after distal gastrectomy for gastric cancer: a meta-analysis. World J Gastroenterol. 2018;24(24):2628-39.

13. Jian-Cheng T, Bo Z, Jian F, Liang Z. Delta-shaped gastroduodenostomy in fully laparoscopic distal gastrectomy: a retrospective study. Medicine (Baltimore). 2015;94(28):e1153.

14. Shinohara T, Kawano S, Tanaka Y, Fujisaki M, Watanabe A, Yamamoto K, et al. Comparison of the cost and outcomes following totally laparoscopic and laparoscopy-assisted distal gastrectomies for gastric cancer: a single-institution comparison. Surg Endosc. 2016;30(8):3573-81.

15. Wang Y, Li Z, Shan F, Zhang L, Li S, Jia Y, et al. Comparison of the safety and the costs between laparoscopic assisted or totally laparoscopic uncut Roux-en-Y and Billroth II(+Braun reconstruction-a single center prospective cohort study. Chin J Gastointest Surg. 2018;21(3):312-7.

16. Sun X, Qiu H, Fei K, Xu L, Lu J, Zhang G, et al. Comparison of intra-abdominal infection between intracorporeal anastomosis and extracorporeal anastomosis in patients undergoing laparoscopic right hemicolectomy. Chin J Gastointest Surg. 2017;20(8):891-5.

17. Kinoshita T, Kanehira E, Matsuda M, Okazumi S, Katoh R. Effectiveness of a team participation training course for laparoscopy-assisted gastrectomy. Surg Endosc. 2010;24(3):561-6.

\section{Publisher's Note}

Springer Nature remains neutral with regard to jurisdictional claims in published maps and institutional affiliations.
Ready to submit your research? Choose BMC and benefit from:

- fast, convenient online submission

- thorough peer review by experienced researchers in your field

- rapid publication on acceptance

- support for research data, including large and complex data types

- gold Open Access which fosters wider collaboration and increased citations

- maximum visibility for your research: over $100 \mathrm{M}$ website views per year

At BMC, research is always in progress.

Learn more biomedcentral.com/submissions 